\title{
APPLICATION OF TAGGED NEUTRON TECHNOLOGY IN RESEARCH, INDUSTRY AND HOMELAND SECURITY
}

\author{
Yuri Barmakov, Viacheslav Batyaev, Evgeniy Bogolyubov, Alexander Gavryuchenkov, \\ Maxim Karetnikov*, Dmitri Yurkov, Valentine Ryzhkov
}

\author{
Dukhov Research Institute of Automatics (VNIIA), Moscow, Russia
}

\begin{abstract}
Each $14 \mathrm{MeV}$ neutron of T(d,n)He4 reaction is accompanied (tagged) by $3.5 \mathrm{MeV}$ alpha-particle emitted in the opposite direction. A position- and time-sensitive alpha-detector measures time and coordinates of the associated alpha-particle for determining the time and direction of the neutron escape. A spectrum of gamma rays emitted as a result of the interaction of tagged neutrons with nuclei allows the identification of the chemical composition of the irradiated object. The recording of alpha-gamma coincidences within a very narrow time window provides the possibility of background suppression by means of spatial and time discrimination of events. The Nanosecond Tagged Neutron Technology (NTNT) based on this principle has great potentialities in various fields, such as the study of neutron scattering, revision of neutron differential cross-sections, measuring the chemical composition of ores in mining industry, the detection of explosives, mines, drugs and chemical warfare agents, etc. The Dukhov Research Institute of Automatics produces a complete set of NTNT equipment, including neutron generators with built-in alpha-detectors, electronic hardware for alpha-gamma coincidence data acquisition, mobile and stationary NTNT systems for various research, industrial and homeland security applications.
\end{abstract}

Key words: Neutron analysis, tagged neutron, gamma rays, neutron generator, explosives detection, homeland security, diamond

DOI: $10.21175 / \operatorname{RadProc} .2016 .11$

Neutron analysis is in great demand in many areas of science and industry. It is due to unique neutron properties, such as high penetrability because of zero electric charge, and selective interaction with nuclei of chemical elements regardless of their chemical or aggregate state.

Neutron analysis is based on the measurement of the secondary gamma-neutron radiation generated as a response to initial neutron radiation. One of the main problems here is a high background, caused mostly by gamma rays generated from interactions of neutrons with the components of the device or the surrounding objects, by-products of the decay of the generated radionuclides, etc. In recent years, the Nanosecond Tagged Neutron Technology (NTNT) has been rapidly progressing. Owing to spatial and time selection of events, the NTNT allows the level of background radiation to be substantially reduced [1].

Figure 1 shows a block diagram of a typical NTNT device. Initial energies and the directions of the neutron and alpha-particle from the $\mathrm{T}(\mathrm{d}, \mathrm{n}) 4 \mathrm{He}$ reaction are unambiguously interrelated. A positionsensitive (multipixel) alpha-detector produces a timestamp ta and records coordinates of the alpha-particle detected, thus providing its motion direction and exit time by means of correction for the alpha-particle velocity. Using this data, one can evaluate the neutron exit time, direction of motion and energy in the direction towards the investigated object, i.e. the neutron can be "tagged" by the associated alphaparticle recorded. This technology is also known as Associated Particle Imaging due to the significance of the associated alpha-particle.

During the inelastic scattering of fast neutrons by nuclei, the energy spectrum of the resultant gamma rays has pronounced signatures, i.e. it is unique for different chemical elements and can be used for their identification. Distance $\mathrm{L}$ from the target of the neutron generator to the point of emission of a gammaquantum, generated during the inelastic scattering of the fast tagged neutron, can be determined by means of measuring the time interval between the detection of the gamma quantum and the alpha particle associated with the neutron. Knowing the distance $\mathrm{L}$ and vector of neutron motion, one can calculate the location of gamma-ray emission. 


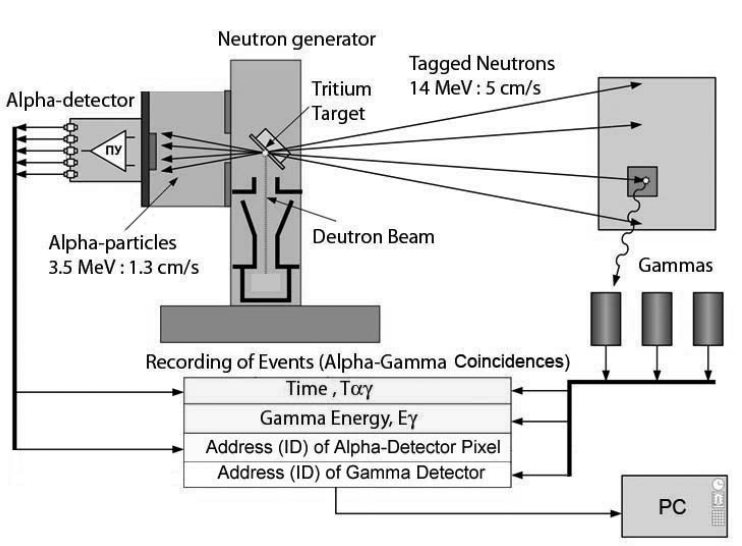

Figure 1. Block diagram of an NTNT device

A key component of NTNT is a neutron generator with a built-in position-sensitive alpha-detector. Currently, off-the-shelf generators of tagged neutrons are manufactured by Thermo Scientific, USA (API-120 series [2]), Adelphi Technology Inc., USA (DT108API) [3], and Dukhov Research Institute of Automatics (VNIIA), Russia (ING-27 series) [4]. All the generators are based on gas-filled neutron tubes. Peak intensity of API-12O is as high as $2 \cdot 10^{7} 1 / \mathrm{s}$. Peak intensity of DT108API and ING-27 is close to $1 \cdot 10^{8} 1 / \mathrm{s}$. The available geometries of the alpha-detector used in ING27 are shown in Figure 2.

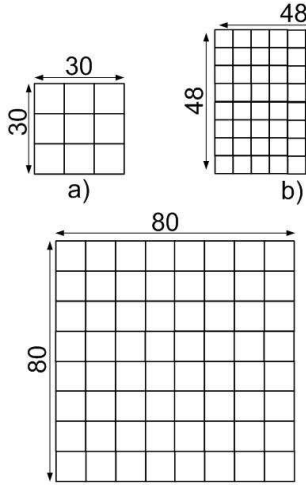

d)

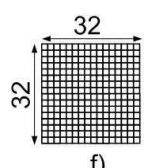

f)
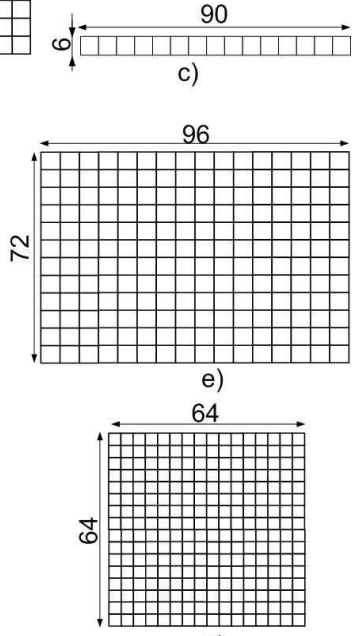

g)
Figure 2. Versions of semiconductor alpha-detectors to be built-in ING-27 neutron generator: a) 3x3 pixels, $10 \times 10 \mathrm{~mm}$ pixel size, b) $8 \times 8$ pixels, $6 \times 6 \mathrm{~mm}$, c) $1 \times 15$ pixels, $6 \times 6 \mathrm{~mm}, \mathrm{~d}$ )

$8 \times 8$ pixels, $10 \times 10 \mathrm{~mm}$, e) $12 \times 16$ pixels, $6 \times 6 \mathrm{~mm}$; f) $16 \times 16$ pixels, 2x2 mm; g) 16x16 pixels, 4X4 mm

The scintillating detectors are used in API-120 and DT108API neutron generators. The scintillator is coupled through an optical plate with a positionsensitive PMT, and the crystal geometry depends on the PMT input window geometry. However, there are few models of position-sensitive PMT that can be used for this task. Hamamatsu $\mathrm{H} 8500$ and $\mathrm{H} 9500$ have the largest size of input window $(52 \times 52 \mathrm{~mm})$ among the available PMT. The assembly of several position- sensitive PMT at the input flange of the neutron generator to increase the sensitive area of the alphadetector heavily complicates the problem.

VNIIA produces specialized silicon and GaAs semiconductor-type detectors for NTNT neutron generators (ING-27). They have certain technological and economical advantages over scintillating alphadetectors, providing at the same time close basic operating parameters (radiation hardness, energy and time resolution). It makes it possible to assemble any reasonable configuration (Figure 2) of the semiconductor alpha-detector limited only by the geometry of the neutron generator flange.

A tagged neutron beam profile (Figure 3) that defines NTNT angular resolution depends on pixel size $\mathrm{S}$, diameter of deuteron beam spot on the target, and distance $\mathrm{D}$ between the target and the pixel. The limiting angular resolution is determined by chromatic and geometric ion-optic aberrations, effects of alphaparticle scattering in the tritium target and neutron scattering in the target holder; and in case of ING-27 it is close to $2-3^{\circ}[5]$.

Basic criterion for selection of useful events is the presence of signals from the alpha- and gammadetectors in a specified time window (around several dozen ns), and the absence of overlapping signals. Nonorganic (NaI(Tl), BGO, LYSO, LaBr3) scintillating gamma-detectors are commonly used for gamma-rays recording. For the neutron generator intensity of the value of $10^{8} 1 / \mathrm{s}$, the total counting rate of alpha- and gamma-detector can exceed $10^{6} 1 / \mathrm{s}$ and $10^{5} 1 / \mathrm{s}$, respectively.

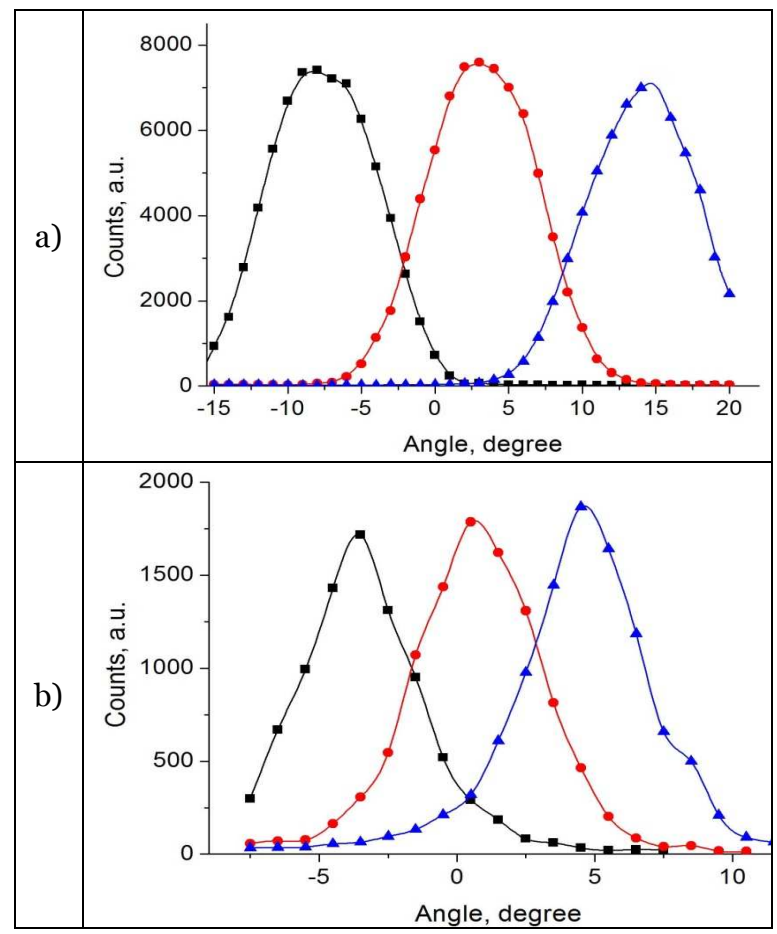

Figure 3. Angular distribution of tagged neutrons corresponding to a) $\mathrm{D}=57 \mathrm{~mm}$ and $\mathrm{S}=10 \times 10 \mathrm{~mm}$,

b) $\mathrm{D}=100 \mathrm{~mm}$ and $\mathrm{S}=6 \times 6 \mathrm{~mm}$. The neighboring pixels are marked by $\bullet, \boldsymbol{\Lambda}, \boldsymbol{\square}$ symbols

The processing of such data stream without the loss and distortion of information is a challenge to the NTNT application. At present, there are two 
approaches to the problem of processing signals from NTNT devices: (1) signal processing by analog frontend electronics, preliminary online selection of pulses by hardware and transmission of only useful events to a PC, and (2) complete digitization of signals from all detectors, and the data stream transmission to an intermediate computer for subsequent processing.

The NTNT devices implementing digitization of signals from all the detectors have a sampling period as low as 7-10 ns. Very short signals from the detectors should be extended in time up to several dozen ns to provide significant number of samples for time and amplitude measurements. It may limit the maximum signal rate. A comparison of digital and analog electronics demonstrates that systems based on analog electronics provide more accurate and stable timing, higher maximum rate of processed events, and lower power consumption.

A special-purpose data acquisition system for the NTNT, based on the principles of analog signal processing, has been under development at VNIIA. To date, several versions of the equipment for mobile and stationary systems differing in dimensions, interface of communication with the PC (USB, RS-485, wireless), and number of channels for recording signals from alpha- and gamma-detectors are developed. They provide an accurate and stable measurement of parameters of alpha-gamma coincidences. The achieved time resolution is as low as $1.0-1.2 \mathrm{~ns}$ for fast LYSO and LaBr3 detectors, and 1.5 - $3.0 \mathrm{~ns}$ for relatively slow $\mathrm{NaI}(\mathrm{Tl})$ and $\mathrm{BGO}$ gamma-detectors [1].

The first practical application of the NTNT was the calibration of neutron detectors [6] in 1963. As far as the NTNT provides "pure" flux of $14 \mathrm{MeV}$ neutrons, it is used nowadays in thermonuclear research for the calibration of neutron detectors and measuring gamma-detectors response to $14 \mathrm{MeV}$ neutrons.

NTNT is a very convenient tool for the study of fast neutrons interaction with different nuclei. It was used for measuring angle correlations of products of inelastic scattering of fast neutrons in numerous works, e.g. [5, 7]. Currently, a cycle of experiments for study $\left(n, n^{\prime} \gamma\right)$ and $\left(n, 2 n^{\prime}\right)$ reactions is run at TANGRA setup [8] (Figure 4) in the Joint Institute for Nuclear Research (JINR), Dubna, Russia. The results of the experiments are essential for nuclear physics and astrophysics.

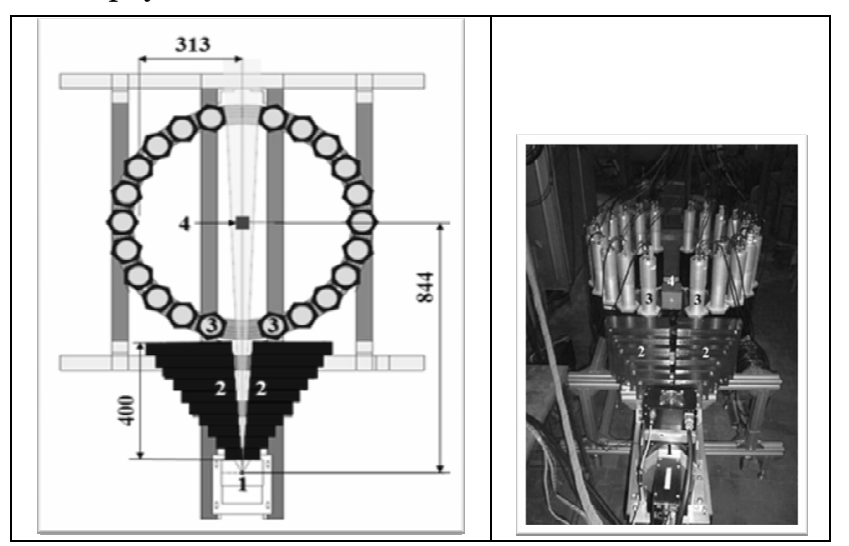

Figure 4. Geometry (on the left, dimensions in $\mathrm{mm}$ ) and appearance (on the right) of TANGRA setup. 1 - ING-27 neutron generator, 2- shielding, 3- scintillating gammadetectors, 4- investigated object
Based on gamma-ray energy and emission location obtained as the result of the acquired data processing, a $3 \mathrm{D}$ map of chemical composition of the interrogated object can be generated. A spatial resolution is determined by the angular resolution of the alphadetector and time resolution of alpha-gamma coincidences. It can be as low as $5-8 \mathrm{~cm}$ along the tagged neutron beam and $2-3 \mathrm{~cm}$ in transverse direction. The NTNT capability to measure gammaspectrum at any specific point of the interrogated volume and high penetrability of fast neutrons and gamma-rays make it possible to locate and identify suspicious items in bulk objects or identify items shielded by massive walls or even thick water layer.

In the mining industry, it allows the detection of large (more than 5 karat) diamonds in kimberlite ore before the crushing stage [9]. Figure 5 displays a setup for diamond search, including a generator of tagged neutrons, an array of BGO gamma-detectors, and a shielding protecting the gamma-detectors from direct neutron bombardment. It simply detects excess carbon at a particular point of the kimberlite sample. The carbon spectrum is characterized by $4.44 \mathrm{MeV}$ peak and first escape peak 3.93 MeV (Figure 6). These lines are absent in the spectrum of kimberlite.

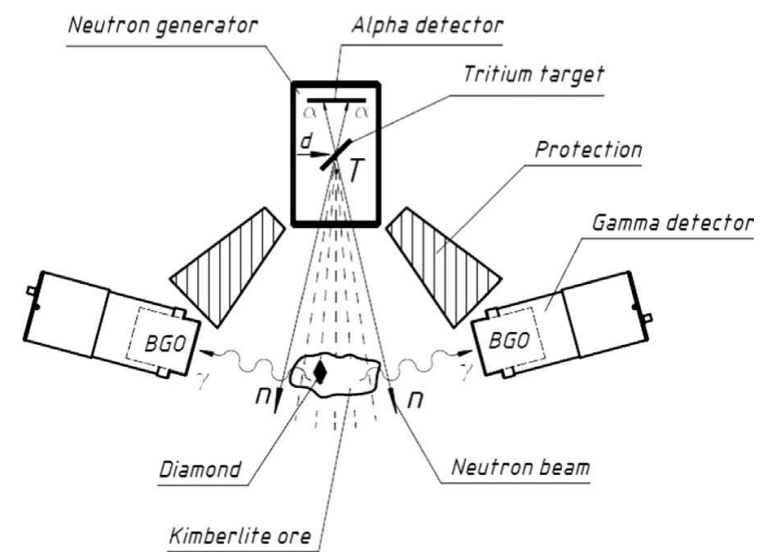

Figure 5. A setup for diamond search

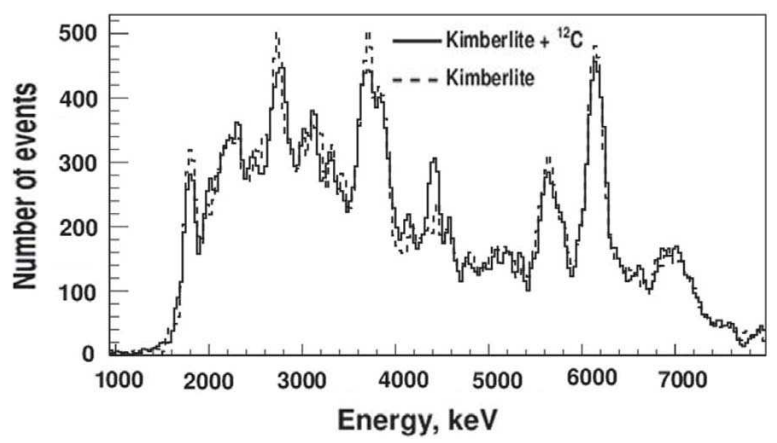

Figure 6. Spectra of gamma-rays from kimberlite (dashed line) and kimberlite with diamond insertions (solid line)

The NTNT can be also used in art and mural restoration when a piece of art is covered by thick layers of different nature (plaster, posterior painting, etc.). The possibility to detect and locate, using the NTNT, chemical elements such as copper, lead, and sulfur that clearly indicate the presence of gesso and pigments was experimentally demonstrated [10]. 
And the most promising applications of the NTNT are certainly connected with the remote detection of explosives. Practically all explosives feature a higher (more than several times) nitrogen concentration compared to harmless nitrogenated materials such as wool, polyurethane, and nylon. Moreover, concentration ratios of carbon, nitrogen, and oxygen nuclei in high explosives are within rather narrow range:

$\mathrm{O} /(\mathrm{C}+\mathrm{N}+\mathrm{O}): 0.3-0.7$

$\mathrm{C} /(\mathrm{C}+\mathrm{N}+\mathrm{O}): 0.15-0.55$

$\mathrm{N} /(\mathrm{C}+\mathrm{N}+\mathrm{O}): 0.1-0.4$.

The NTNT capability to detect key chemical elements $\mathrm{C}, \mathrm{N}$, and $\mathrm{O}$ increases the probability of explosives detection compared to technologies based only on nitrogen determination. In addition, when a substance is identified based on several elements, it is possible to normalize their concentration (for example calculate $\mathrm{C} /(\mathrm{C}+\mathrm{N}+\mathrm{O}), \quad \mathrm{N} /(\mathrm{C}+\mathrm{N}+\mathrm{O}), \quad \mathrm{O} /(\mathrm{C}+\mathrm{N}+\mathrm{O})$ ratios), which is very important in case of strong neutron and gamma-ray attenuation in bulk objects.

An experimental and theoretical study of NTNT devices for high explosives detection demonstrated the possibility of explosives identification against the background of innocent materials that can be frequently met at the passenger luggage and that are rather close to explosives by density and average atomic number (paper, clothes, foodstuff). An example of the experimental device is given in Figure 7 [11]. It includes the scintillation LYSO gamma detector of $\varnothing_{76 \times 76} \mathrm{~mm}$ crystal size, ING-27 neutron with 9-pixel alpha detector, tungsten bar protecting the gamma detector against direct neutron exposure.

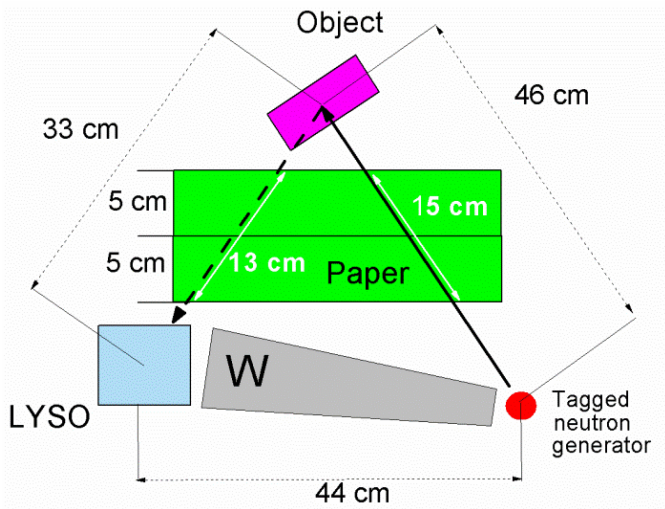

Figure 7. Configuration of the experiment

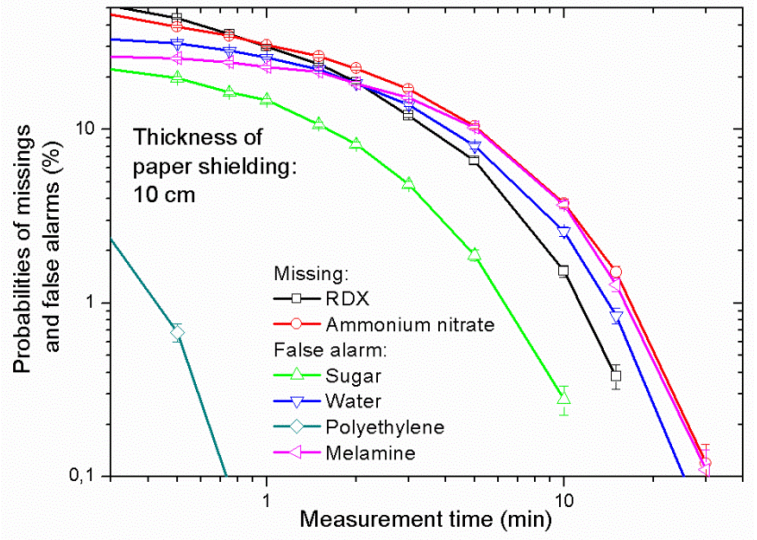

Figure 8. Probability of missing the explosives and false alarm probability caused by innocent materials vs. measurement time
High explosives were imitated by specially made $300 \mathrm{~g}$ simulators of RDX, tetryl, and TNT of appropriate densities and ratios of chemical elements. Polyethylene, melamine, sugar, and water in the amount of $300-500 \mathrm{~g}$ were used as innocent materials. Paper bundles with the total thickness of 10 $\mathrm{cm}$ were placed directly in front of the object attenuated both the initial $14 \mathrm{MeV}$ neutrons and response gamma-rays from the irradiated object. Special algorithms described in detail in [11] were used for processing the experimental data and prediction of detection and false alarm probabilities. Results given in Figure 8 demonstrate that, even in such a hard case, the explosives can be reliably detected for the reasonable time of measurements.

Taking into account a diversity of threats associated with explosives, a variety of explosive and bomb configurations, as well as the types of investigated objects (from mail parcels and mobile phones to cargo containers and vehicles), different NTNT systems for explosives detection were suggested. They can be roughly divided into several types:

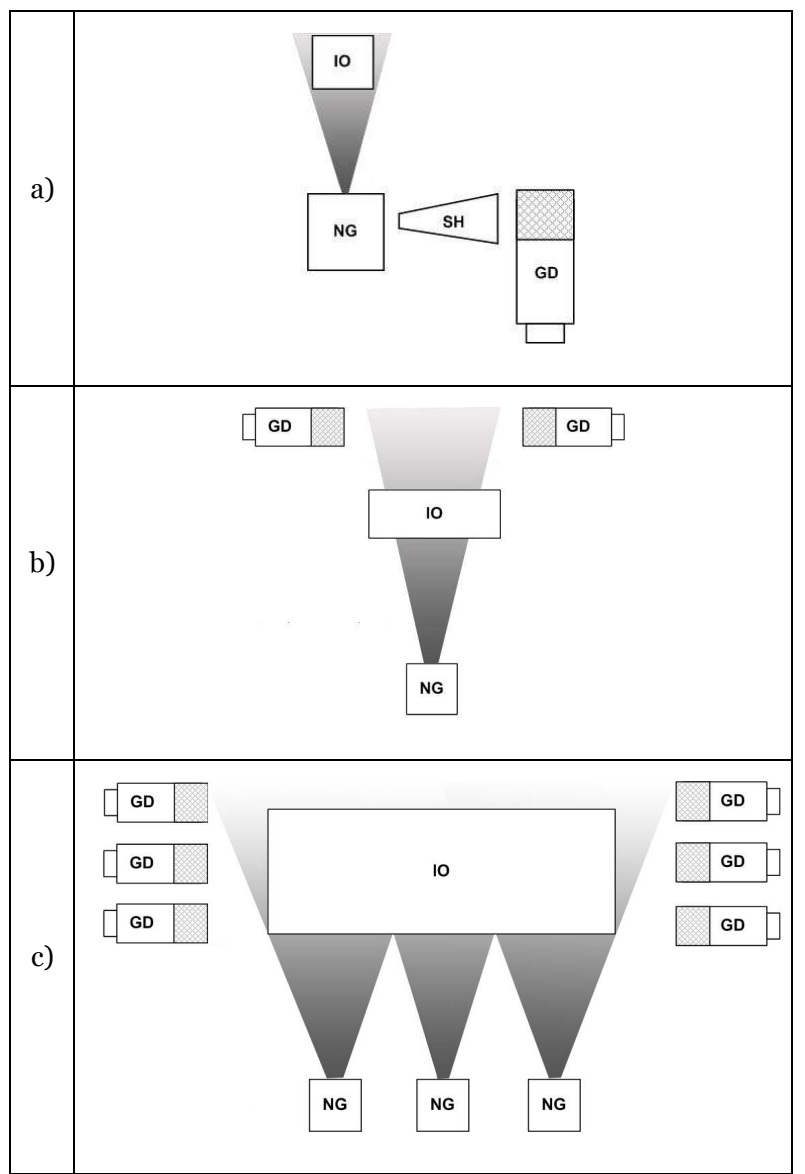

Figure 9. NTNT systems for explosives detection: a) Mobile system, b) Stationary system for baggage and hand luggage control, c) Stationary systems for control of cargo containers and vehicles. IO -investigated object, $\mathrm{SH}$ - shielding, NG neutron generator, GD - gamma-detectors

> Mobile systems for detecting mines, for the inspection of unattended and suspicious objects, and etc. The sounding by tagged neutrons and measurement of induced gamma-rays are carried out from one side (Figure 7, 9a). The mobile systems include 1 to 4 gamma-detectors; a minimal detectable 
mass of explosives is as low as dozens grams. Over a hundred of these systems based on 9-pixel ING-27 neutron generator are currently used in the Moscow Metro for the control of suspicious objects [12].

$>$ Stationary systems for baggage and hand luggage control. The investigated object is located between the neutron generator and gamma-detectors, the quantity of which varies from 6 to 12 (Figure 9b). A minimal detectable mass of explosives is several hundred grams.

$>$ Stationary systems for the control of cargo containers and vehicles. Gamma-detectors are aside the tagged neutron beam axis (Figure 9c). A total number of gamma-detectors is several dozen pieces. Accuracy of inspection parameters can be improved by means of usage of several neutron generators and/or a movable neutron generator that is set in motion along a rail by a step motor. Minimal detectable mass of explosives depends on the explosive location and shielding capabilities of surrounding material, and starts from several kilograms.

It should be noted that for any scenario of NTNT application radioactivation of an interrogated object is low, and residual activity is harmless both for the object carrier and for personnel. There are practically no other potential dangerous effects, such as degradation of semiconductor or optical devices, radiochemical deterioration of materials, and etc. Thus, the NTNT control meets radiation safety standards even in case of the repeated irradiation of the same objects during a year. In some cases, the NTNT can be used even for neutron analysis of living beings. For example, what was suggested and proven from the point of view of radiation safety was the invivo control of fat, protein, and water during the growth process of lambs [13].

There are also other gamma-neutron technologies of similar sensitivity that are based on high-energy (several $\mathrm{MeV}$ ) deuteron accelerators with nanosecond pulses [14] (PFNA, NRR, FTNA, FSNA). Their main disadvantages are high maintenance cost and capital expenditures, considerable weight, dimensions, and associated problems concerning their integration in the existing control systems. The NTNT system weight and dimensions are close to that of X-ray systems intended for the control of similar objects. The NTNT uses the existing technological and elemental base. Currently there are no physical and operating limitations revealed that hinder the wide application of the NTNT equipment to research, industry and homeland security.

\section{REFERENCES}

1. M. Karetnikov et al., "Efficiency of Background Suppression by Tagged Neutron Technology," Nucl. Instr. Meth. Phys. Res. B, vol. 261, no. 1-2, pp. 307-310, Aug. 2007

2. D.L. Chichester, M. Lemchak and J.D. Simpson, "The Api 120: A Portable Neutron Generator for the Associated Particle Technique," Nucl. Instr. Meth. Phys. Res. B, vol. 241, no. 1-4, pp. 753-758, Dec. 2005

3. DT110-14 MeV Neutron Generator, Adelphi Technology Inc., Redwood City (CA), USA, 2015

Retrieved from: http://www.adelphitech.com/products/dt110.html

4. T. Khasaev, A. Golubev, S. Korotkov, "Compact Neutron Source ING-27 for Elemental Analysis Technique," in Proceed. Int. Conf. Portab. Neut. Gen. Techn. Basis, Moskow, Russia, 2012, pp. 60-67

5. M.D. Karetnikov et al., "Angular Correlations in Detection of $\alpha-\gamma$ Coincidences in the Nanosecond Tagged Neutron Technology," Instr. Exper. Techn., vol. 52, no. 4, pp. 497-501, July 2009

6. J.D.L.H. Wood, "A Sealed-Off $14 \mathrm{MeV}$ Neutron Source Incorporating a Solid State Alpha-Particle Detector," Nucl. Instr. Meth., pp. 49-52, Jan.-Feb. 1963

7. D. Spaargaren and C.C. Jonker, "Angular Correlations in Inelastic Neutron Scattering by Carbon at $15.0 \mathrm{MeV}$," Nucl. Phys. A, vol. 161, no. 2, pp. 354-374, Feb. 1971

8. I.N. Ruskov et al., "TANGRA-Setup for the Investigation of Nuclear Fission Induced by $14.1 \mathrm{MeV}$ Neutrons," Phys. Proced., vol.64, pp. 163-170, 2015

9. V.Yu. Alexakhin et al, "Detection of Diamonds in Kimberlite by the Tagged Neutron Method," Nucl. Instr. Meth. Phys. Res. A, vol. 785, pp. 9-13, June 2015

10. A.V. Kuznetsov et al., "Nanosecond Neutron Analysis for the Search of the Lost Leonardo's Masterpiece, the Battle of Anghiari," Nucl. Instr. Meth. Phys. Res. B, vol. 267, no. 23-24, pp. 3694-3697, Dec. 2009

11. V.F. Batyaev, S.G. Belichenko, R.R. Bestaev, A.V. Gavryuchenkov, M.D. Karetnikov, "Tagged Neutron Capabilities for Detecting Hidden Explosives," in Proceed. SPIE, vol.9454, May 2015

12. Neutron Generators Video, VNIIA. Retrieved from: http://vniia.ru/eng/ng/docs/dosmotr eng.mp4.

13. S. Mitra, J.E. Wolff, R. Garrett and C.W. Peters, "Whole Body Measurement of $\mathrm{C}, \mathrm{N}$ and $\mathrm{O}$ using $14 \mathrm{MeV}$ Neutrons and the Associated Particle Time-of-Flight Technique," Asia Pac. J. Clin. Nutr., vol. 4, no. 1, pp. 187-189, 1995

14. T. Gozani "The Role of Neutron Based Inspection Techniques in the Post 9/11/o1 Era," Nucl. Instr. Meth. Phys. Res. B, vol. 213, pp. 460-463, Jan. 2004 